\title{
Enhanced Recovery After Surgery (ERAS) in Gynaecology: a meta-analysis
}

\author{
Zixuan Song ${ }^{1}$, Yizi Wang ${ }^{1}$, Dandan Zhang ${ }^{1}$, and Yangzi Zhou ${ }^{1}$ \\ ${ }^{1}$ Shengjing Hospital of China Medical University
}

August 23, 2020

\begin{abstract}
Background Enhanced recovery after surgery (ERAS), which originated in colorectal surgery, has been implemented in different types of surgery, but its effectiveness in gynaecologic surgery remains to be determined. Objectives A meta-analysis was conducted on the existing studies to compare the effects of ERAS pathways and routine management on the outcomes of gynaecological surgeries. Search Strategy The main database was retrieved from inception to 24 July 2020 using the term 'enhanced recovery after surgery' and its changes, as well as search restrictions for related study designs. Selection Criteria Studies whose number of ERAS pathways projects implemented by the ERAS group but not by the conventional surgery group was no less than four were included. Outcomes included length of hospital stay, complications, and so on. Data Collection and Analysis According to the accurate data extraction table, the fixed or random effects model was used to summarize. Main Results The length of hospital stay (LOS) situation of the ERAS group was significantly shorter than that of the control group, including the shortening of primary $\operatorname{LOS}(\mathrm{SMD}=-0.681,95 \% \mathrm{CI}:-0.844-0.517, \mathrm{P}<0.01)$ and total $\mathrm{LOS}(\mathrm{SMD}=-0.312,95 \% \mathrm{CI}$ : $-0.551-0.073, \mathrm{P}=0.011$ ), as well as the increase of the number of people reaching the target $\operatorname{LOS}$ (OR=4.899, 95\% CI: 3.825$6.276, \mathrm{P}<0.01)$. Conclusions The available evidence suggests that ERAS pathways may reduce the length of postoperative hospital stay in gynaecologic surgery, without increasing complications, while shortening the time needed for postoperative functional recovery. Keywords Enhanced recovery after surgery, ERAS, gynaecology
\end{abstract}

Enhanced Recovery After Surgery (ERAS) in Gynaecology: a meta-analysis

Co-authors Zixuan Song, Yizi Wang, Dandan Zhang, Yangzi Zhou

Address Department of Obstetrics and Gynecology, Shengjing Hospital of China Medical University, Shenyang 110004, People's Republic of China

Corresponding author Yangzi Zhou

Address Department of Obstetrics and Gynecology, Shengjing Hospital of China Medical University, Shenyang 110004, People's Republic of China

Tel 86-18940258515

E-mail zhouyz@sj-hospital.org

Running Head ERAS in Gynaecology

Abstract

Background Enhanced recovery after surgery (ERAS), which originated in colorectal surgery, has been implemented in different types of surgery, but its effectiveness in gynaecologic surgery remains to be determined.

Objectives A meta-analysis was conducted on the existing studies to compare the effects of ERAS pathways and routine management on the outcomes of gynaecological surgeries. 
Search Strategy The main database was retrieved from inception to 24 July 2020 using the term 'enhanced recovery after surgery' and its changes, as well as search restrictions for related study designs.

Selection Criteria Studies whose number of ERAS pathways projects implemented by the ERAS group but not by the conventional surgery group was no less than four were included. Outcomes included length of hospital stay, complications, and so on.

Data Collection and Analysis According to the accurate data extraction table, the fixed or random effects model was used to summarize.

Main Results The length of hospital stay (LOS) situation of the ERAS group was significantly shorter than that of the control group, including the shortening of primary LOS (SMD=-0.681, 95\% CI: -0.844-0.517, $\mathrm{P}<0.01$ ) and total LOS (SMD=-0.312, 95\% CI: $-0.551-0.073, \mathrm{P}=0.011$ ), as well as the increase of the number of people reaching the target LOS $(\mathrm{OR}=4.899$, 95\% CI: 3.825-6.276, $\mathrm{P}<0.01)$.

Conclusions The available evidence suggests that ERAS pathways may reduce the length of postoperative hospital stay in gynaecologic surgery, without increasing complications, while shortening the time needed for postoperative functional recovery.

Keywords Enhanced recovery after surgery, ERAS, gynaecology

Funding No funding sources.

Tweetable Abstract

ERAS pathways may reduce the length of postoperative hospital stay in gynaecologic surgery, without increasing complications.

Introduction

In 2003, Kehlet and Dahl proposed a new theory, Enhanced Recovery after Surgery (ERAS) ${ }^{1}$. This theory changes the surgical management model from the original "wait and see" model to a more positive model. ERAS pathways were first applied in colorectal surgery and later extended to other surgical specialties ${ }^{2-5}$. In the process of application, ERAS pathways have obvious advantages and thus are promoted in gynaecology. In 2016, the first edition of guidance on ERAS pathways of gynaecology was released, which effectively promoted the development of ERAS pathways in gynaecology 6,7 .

The success of ERAS pathways is not based on a single clinical practice intervention but represents a multi-model approach to perioperative rehabilitation. ERAS is based on a multidisciplinary team working together, relying not only on gynaecologists but also on the active participation of anaesthesiologists, nurses, pharmacists and dietitians, as well as patients. Prior to surgery, patient education and counselling is included, intestinal preparation is avoided, and an unstrict fasting water strategy is implemented. During surgery, PONV prevention is included, a standard anaesthesia regimen is performed, body temperature is maintained, and fluid volume is balanced. After surgery, ERAS includes multimodal labour, early oral administration, and early mobilization. Examples of ERAS pathways measures in gynaecological surgery are shown in Table $1^{6-8}$. ERAS has been widely popularized in gynaecology over the past few years, and some studies have reported relevant results. We summarized these studies and conducted a meta-analysis to compare the different influences of ERAS and non-ERAS on postoperative outcomes of gynaecological surgeries.

Methods

Search strategy

From the date of publication until 24 July 2020, literature was searched and confirmed by PubMed, Embase and Cochrane Central Controlled Trial Register databases. A predefined search policy was used (Appendix S1). There were no language restrictions on the references, including manuscripts and reviews. The abstracts of the initial search were evaluated to determine whether they were compatible with the research problem. Conference summary books were used to identify potentially eligible studies. 
Qualification standard

Inclusion criteria were as follows. (i) Laparotomy, laparoscopy or pelvic floor gynaecological surgery was performed for benign or malignant gynaecological diseases. (ii) The project of ERAS pathways is described. If there is a description of the number of specific participants in a single project of ERAS pathways, more than $50 \%$ of the participants consider the implementation of the project. (iii) The number of ERAS pathways projects implemented by the ERAS group but not by the conventional surgery group is no less than four. The selection of this threshold is consistent with other system reviews ${ }^{9}$. (iv) Contains at least one predefined primary result. (v) The control group adopted the conventional perioperative nursing mode. (vi) Information on patient characteristics and potential confounding factors should be provided. (vii) If the patient overlapped between the research, only the latest information is included.

Quality assessment

The quality evaluation was conducted independently by two authors (Zixuan Song and Dandan Zhang). Any differences were discussed with Dr Yangzi Zhou before reaching an agreement. For all studies, we used the Downs and Black checklist for quality assessment ${ }^{10}$.

Data extraction

The data were extracted independently by two authors (Zixuan Song and Yizi Wang). If there was any disagreement, Dr. Yangzi Zhou made a decision. The first author of the included study, year of publication, sample size, participation of ERAS pathways project, surgical method, treatment response, and follow-up time were recorded.

Statistical analysis

The meta-analysis was performed using Stata 13.0 software. The group data were summarized by descriptive statistics. We estimated a $\mathrm{p}$ value and $95 \% \mathrm{CI}$ through a fixed or random effects model ${ }^{11}$. Inter-study heterogeneity was quantified by the $\mathrm{I}^{2}$ metric and $\mathrm{P}$ value through the $\chi^{2}$ test, $\mathrm{I}^{2}[?] 50 \%$ and $\mathrm{P}<0.10$ indicated greater heterogeneity ${ }^{12}, 13$. We performed a subgroup analysis of the main results. Begg's ${ }^{14}$ and Egger's ${ }^{15}$ regression were used to assess publication bias of the main results. If Begg's and Egger's regression suggested publication bias, trim and fill analysis ${ }^{16}$ was performed.

Results

Identification and selection of eligible studies

A flowchart of the literature search is depicted in Fig. 1. An initial search turned up 1862 articles. After eliminating duplicates, 1825 articles were reviewed for title and summary, of which 1717 articles were excluded from further analysis because of their apparent irrelevance. After full-text evaluation of the remaining 108 articles, 30 articles ware preliminarily consistent with the study. Through the analysis of the implementation of ERAS pathways project(Appendix S2), 27 articles were eventually included in this meta-analysis.

Characteristics of the research

The descriptive characteristics of the included studies are shown in Table 2. Three RCTs ${ }^{17-19}$ and 24 Non$\mathrm{RCTs}^{20-43}$ were included in this meta-analysis, involving a total of 6344 patients.

Methodological quality of included studies

The Downs and Black checklist results of all the studies are shown in Appendix S3. All the studies scored from 16-20 points, which represents 'moderate quality'.

Meta-analysis

The primary and secondary results of this study were obtained directly from the included literature or were estimated indirectly from the literature data. The primary outcomes of this study were postoperative length of stay (LOS), including primary LOS (PLOS), total LOS (TLOS) and the number of people 
discharged from the hospital before the target length of stay (tLOS). TLOS represents total LOS within 30 days of surgery, including readmission days. Twenty-four studies ${ }^{17-23,25-36,38-42}$ reported PLOS, five studies $^{20,23,24,34,37}$ reported TLOS, and eight studies $24,26,28,29,32,36,40,43$ reported TLOS. Compared with the control group, the PLOS of ERAS group was significantly shortened (SMD=-0.681, 95\% CI: 0.844-0.517, $\mathrm{P}<0.01)$, TLOS was significantly shortened(SMD=-0.312, 95\% CI: $-0.551-0.073, \mathrm{P}=0.011)$, and tLOS increased significantly $(\mathrm{OR}=4.899,95 \% \mathrm{CI}: 3.825-6.276, \mathrm{P}<0.01)$. The primary outcomes are shown in Fig. 2 .

We assessed publication bias for primary results. PLOS [Begg's test, $\mathrm{P}=0.006$ (Fig.3A); Egger's test, $\mathrm{P}=0.006$ (Fig.3B)] may have a publication bias, and no significant publication bias was observed in TLOS [Begg's test, $\mathrm{P}=0.086$ (Fig.3C); Egger's test, $\mathrm{P}=0.075$ (Fig.3D)] and tLOS [Begg's test, $\mathrm{P}=0.063$ (Fig.3E); Egger's test, $\mathrm{P}=0.148$ (Fig.3F)]. We did a trim and fill analysis of PLO and found that the PLOS of the ERAS group was still significantly shorter than that of the control group after filling in the two results[SMD=-0.746, 95\% CI: -0.926-0.566, P<0.01(Fig.4)]. Our study suggests that PLOS publication bias can be ignored.

In this study, we performed a subgroup analysis of PLOS based on disease and surgical modalities. The PLOS of the ERAS group was significantly shorter than that of the control group, whether benign(SMD=0.894, 95\% CI: $-1.076-0.711, \mathrm{P}<0.01)$ or malignant(SMD=-0.502, 95\% CI: $-0.639-0.365, \mathrm{P}<0.01)$. Compared with the control group, the PLOS of the ERAS group was significantly shortened for minimally invasive surgery $(\mathrm{SMD}=-0.713,95 \% \mathrm{CI}:-1.220-0.206, \mathrm{P}<0.01)$, open surgery $(\mathrm{SMD}=-0.488,95 \% \mathrm{CI}:-0.628-0.348$, $\mathrm{P}<0.01)$ and pelvic floor surgery $(\mathrm{SMD}=-1.203,95 \% \mathrm{CI}:-1.532-0.874, \mathrm{P}<0.01)$. The subgroup analysis results are shown in Table 3.

Secondary outcomes are targeted at complications, perioperative conditions, postoperative motion, restoration of digestive function, pain and costs. For complications, compared with the control group, the number of patients with complications in the ERAS group was significantly reduced $(\mathrm{OR}=0.664,95 \% \mathrm{CI}: 0.481-0.917$, $\mathrm{P}=0.013)$, while there was no significant difference in death within 30 days $(\mathrm{OR}=0.758,95 \% \mathrm{CI}: 0.330-$ $1.743, \mathrm{P}=0.515)$, readmission $(\mathrm{OR}=0.973,95 \% \mathrm{CI}: 0.799-1.184, \mathrm{P}=0.781)$ or reoperations $(\mathrm{OR}=0.701,95 \%$ $\mathrm{CI}: 0.244-2.014, \mathrm{P}=0.51)$. For perioperative conditions, time of surgery(SMD=-0.047, 95\% CI: $-0.163-0.069$, $\mathrm{P}=0.429)$ and estimated blood $\operatorname{loss}(\mathrm{SMD}=-0.074,95 \% \mathrm{CI}:-0.151-0.004, \mathrm{P}=0.063)$ showed no difference between the ERAS group and the control group. While perioperative intravenous fluids $(\mathrm{SMD}=-1.535,95 \%$ CI: $-2.370-0.700, \mathrm{P}<0.01)$ significantly decreased in the ERAS group. For postoperative motion, compared with the control group, the ERAS group saw a significant increase in the number of people who could get out of bed on the first postoperative day $(\mathrm{OR}=4.597,95 \% \mathrm{CI}: 2.331,9.064, \mathrm{P}<0.01)$, while the time to first walk $(\mathrm{SMD}=-2.717,95 \% \mathrm{CI}:-4.244-1.189, \mathrm{P}<0.01)$ was significantly shortened. For the restoration of digestive function, compared with the control group, the time to start of liquid $\operatorname{diet}(\mathrm{SMD}=-3.599,95 \% \mathrm{CI}$ : $-4.272-2.925, \mathrm{P}<0.01)$, time to start of semi-solid $\operatorname{diet}(\mathrm{SMD}=-4.253,95 \% \mathrm{CI}:-5.005-3.501, \mathrm{P}<0.01)$, time to eating solid food(SMD=-1.302, 95\% CI: $-1.908-0.696, \mathrm{P}<0.01)$, time to first flatus $(\mathrm{SMD}=-2.194,95 \%$ CI: $-3.777-0.610, \mathrm{P}<0.01)$ and time to first defecation(SMD=-1.400, 95\% CI: $-1.741-1.060, \mathrm{P}<0.01)$ of the ERAS group were significantly shortened. In terms of pain, the amount of morphine equivalent $(\mathrm{SMD}=-$ $0.786,95 \% \mathrm{CI}:-1.391-0.180, \mathrm{P}=0.011)$ was significantly reduced in the ERAS group, with no increase in pain scores. Specifically, in the ERAS group, the pain score on the day of surgery $(\mathrm{SMD}=-0.178,95 \% \mathrm{CI}$ : $-0.290-0.066, \mathrm{P}<0.01)$ was significantly reduced, while the highest pain score $(\mathrm{SMD}=0.110,95 \% \mathrm{CI}:-0.009-$ $0.229, \mathrm{P}=0.071)$, the pain score on the first day after surgery $(\mathrm{SMD}=-0.089,95 \% \mathrm{CI}:-0.197,0.020, \mathrm{P}=0.108)$ and the pain score on the second day after surgery $(\mathrm{SMD}=0.010,95 \% \mathrm{CI}:-0.191-0.211, \mathrm{P}=0.924)$ were not significantly different. With regard to costs, the ERAS group's costs were significantly lower than the control group $(\mathrm{SMD}=-0.258,95 \% \mathrm{CI}:-0.349-0.167, \mathrm{P}<0.01)$. The secondary outcomes are shown in Table 4 .

Discussion

Main findings

In the included studies, the LOS situation of the ERAS group was significantly shorter than that of the control group, including the shortening of PLOS and TLOS, as well as an increase in the number of people reaching 
the target LOS(tLOS). The PLOS shortening of benign diseases and pelvic floor surgery in the ERAS group was more significant. The ERAS group did not show increased complications, operation time, operation bleeding, pain or cost compared with the control group. The ERAS group showed better postoperative activity and gastrointestinal function recovery than the control group.

Strengths and limitations

Although this study is a meta-analysis based on the data of gynaecology ERAS research in recent years, the present results are comparable to the conclusions of other systematic reviews ${ }^{9,}{ }^{44}$, yet there are still some limitations. First, the research included in the analysis was heterogeneous in design. Our study included only three RCTs and the remaining 24 were non-RCT studies, so there was an inherent bias. In addition, the number of studies was limited, and some data were estimated based on the original data, which reduces the overall research quality and weakens the results of meta-analysis. Therefore, the results of this study need to be interpreted carefully.

Interpretation

ERAS first became popular in Northern Europe, eventually spreading around the world and forming the international multidisciplinary ERAS Society due to its shorter hospital stay, faster functional recovery, decreased opioid use, and increased patient satisfaction ${ }^{29,}{ }^{44-46}$. ERAS pathways include multiple elements to enhanced recovery, all of which are critical to the successful implementation of ERAS pathways ${ }^{23}$. Studies that only contain a small number of ERAS elements cannot reflect the advantages of ERAS. Moreover, the stronger the compliance to the elements, the more obvious the therapeutic effect of ERAS ${ }^{47-50}$. ERAS is not a single theory of a single discipline, but a synthesis of advanced theories of multiple disciplines such as surgery, anaesthesiology, nursing and nutrition in recent years ${ }^{8}$. Therefore, in the actual treatments, doctors sometimes are not familiar with ERAS pathways but are still influenced by some elements of the approach and make partial improvements to traditional treatment methods ${ }^{34}$. Therefore, our study not only pays attention to the implementation of ERAS elements in the ERAS group, but also pays attention to whether ERAS elements were applied in the control group when screening for articles. Those studies with more than four ERAS elements implemented in the ERAS group and not implemented in the control group were finally included in our meta-analysis. By analysing the elements of ERAS pathways in the included study, we found that the degree of implementation of ERAS pathways in different studies was different. Studies ${ }^{51,52}$ have found that ERAS' execution degree was different in different cooperative groups. This difference in the degree of implementation of ERAS pathways is also a challenge for ERAS pathways research.

The lack of high-quality randomized controlled trials (RCTs) is a shortcoming of gynaecology ERAS research $^{53}$. This defect is determined by the ERAS pathways itself. The implementation of ERAS pathways requires not only medical personnel, but also the cooperation of patients. Therefore, subjective factors cannot be completely excluded in the study of ERAS pathways. The three RCTs ${ }^{17-19}$ included in our meta-analysis referred only to the randomization of patients but did not refer to blinded medical personnel or patients. An RCT design for ERAS pathways study also mentions that medical personnel, patients, and family members will not be blinded in the trial due to the procedures differing between groups and physicians who perform the surgery ${ }^{54}$.

A major advantage of ERAS pathways is that the approach shortens $\operatorname{LOS}^{55}$. Our results suggest that both PLOS and TLOS in the ERAS group were significantly lower than those in the control group, which is consistent with the results of existing systematic review studies ${ }^{9}, 44$. But existing systems review studies do not focus on tLOS. As medical developments in recent years have focused on shortening the length of hospital stay, in some medical institutions, even before ERAS pathways was developed, some patients had a very short hospital stay for minimally invasive, pelvic floor surgery and minor open surgery, and could even be discharged on the same day of surgery ${ }^{36,40,43}$. Therefore, ERAS pathways studies of these medical institutions cannot find the advantages of ERAS pathways if the evaluation of their results focuses on the length of hospitalization. As a result, only a few studies $26,28,29,32,36,40,43,56$ have looked at tLOS. For some minor operations, they set the target length of stay in advance and observed the number of people who 
could finally reach the target length of stay. In this study, the tLOS of ERAS group was significantly higher than that of the control group. Therefore, ERAS pathways also has advantages for operations with a short hospital stay.

ERAS is a new theory that has made great changes to traditional theory. Conventional wisdom has been ingrained in surgeons for generations and will take time to change. COVID-19 has caused a massive global outbreak and is currently a major global public health problem, and there is no way to know when the COVID-19 pandemic will stop ${ }^{57,58}$. Reducing the length of time patients spend in the hospital also reduces the risk of contracting CONVID-19. In particular, patients with gynaecologic tumours are more susceptible to infection because malignant tumours and anticancer therapies can lead to immunosuppressive status ${ }^{59}$. Reducing the length of stay would also enable hospital staff and resources to focus on those most in need during this period of global demand. Therefore, it is necessary and urgent to promote ERAS pathways that can reduce hospitalization time and enable patients to recover quickly.

Conclusion

In our study, ERAS pathways significantly shortened the length of hospital stay without increasing complications, operation time, intraoperative blood loss and cost. ERAS pathways also shortened the time needed for postoperative functional recovery and reduced morphine consumption without increasing pain. In the future, our findings will need to be confirmed with large-scale, well-designed, high-quality RCTs.

Disclosure of interests

The authors declare that they have no competing interests.

Contribution to authorship

Conception of the study: Zixuan Song, Yangzi Zhou

Search strategy: Dandan Zhang, Zixuan Song

Designed the statistical analysis plan: Yizi Wang, Dandan Zhang

Drafted the manuscript: Zixuan Song, Yizi Wang

All authors have approved the publication of the manuscript.

Funding

No funding sources.

References

1. Kehlet H, Dahl JB. Anaesthesia, surgery, and challenges in postoperative recovery. Lancet. 2003 Dec 6;362(9399):1921-8.

2. Cerantola Y, Valerio M, Persson B, Jichlinski P, Ljungqvist O, Hubner M, et al. Guidelines for perioperative care after radical cystectomy for bladder cancer: Enhanced Recovery After Surgery (ERAS((R))) society recommendations. Clin Nutr. 2013 Dec;32(6):879-87.

3. Lassen K, Coolsen MM, Slim K, Carli F, de Aguilar-Nascimento JE, Schafer M, et al. Guidelines for perioperative care for pancreaticoduodenectomy: Enhanced Recovery After Surgery (ERAS(R)) Society recommendations. World J Surg. 2013 Feb;37(2):240-58.

4. Mortensen K, Nilsson M, Slim K, Schafer M, Mariette C, Braga M, et al. Consensus guidelines for enhanced recovery after gastrectomy: Enhanced Recovery After Surgery (ERAS(R)) Society recommendations. Br J Surg. 2014 Sep;101(10):1209-29.

5. Nygren J, Thacker J, Carli F, Fearon KCH, Norderval S, Lobo DN, et al. Guidelines for Perioperative Care in Elective Rectal/Pelvic Surgery: Enhanced Recovery After Surgery (ERAS@) Society Recommendations. World Journal of Surgery. 2013 2013/02/01;37(2):285-305. 
6. Nelson G, Altman AD, Nick A, Meyer LA, Ramirez PT, Achtari C, et al. Guidelines for postoperative care in gynecologic/oncology surgery: Enhanced Recovery After Surgery (ERAS@) Society recommendationsPart II. Gynecologic oncology. 2016 Feb;140(2):323-32.

7. Nelson G, Altman AD, Nick A, Meyer LA, Ramirez PT, Achtari C, et al. Guidelines for pre- and intra-operative care in gynecologic/oncology surgery: Enhanced Recovery After Surgery (ERAS@) Society recommendations-Part I. Gynecologic oncology. 2016 Feb;140(2):313-22.

8. Nelson G, Bakkum-Gamez J, Kalogera E, Glaser G, Altman A, Meyer LA, et al. Guidelines for perioperative care in gynecologic/oncology: Enhanced Recovery After Surgery (ERAS) Society recommendations-2019 update. International journal of gynecological cancer : official journal of the International Gynecological Cancer Society. 2019 May;29(4):651-68.

9. de Groot JJA, Ament SMC, Maessen JMC, Dejong CHC, Kleijnen JMP, Slangen BFM. Enhanced recovery pathways in abdominal gynecologic surgery: a systematic review and meta-analysis. Acta Obstetricia et Gynecologica Scandinavica. 2016;95(4):382-95.

10. Downs S, Black N. The feasibility of creating a checklist for the assessment of the methodological quality both of randomised and non-randomised studies of health care interventions. J Journal of epidemiology community health

1998;52(6):377-84.

11. Lau J, Ioannidis JP, Schmid CH. Quantitative synthesis in systematic reviews. Annals of internal medicine. 1997 Nov 1;127(9):820-6.

12. Higgins JP, Thompson SG, Deeks JJ, Altman DG. Measuring inconsistency in meta-analyses. Bmj. 2003 Sep 6;327(7414):557-60.

13. Ioannidis JP, Patsopoulos NA, Evangelou E. Uncertainty in heterogeneity estimates in meta-analyses. Bmj. 2007 Nov 3;335(7626):914-6.

14. Begg CB, Mazumdar M. Operating characteristics of a rank correlation test for publication bias. Biometrics. 1994 Dec;50(4):1088-101.

15. Egger M, Davey Smith G, Schneider M, Minder C. Bias in meta-analysis detected by a simple, graphical test. Bmj. 1997 Sep 13;315(7109):629-34.

16. Duval S, Tweedie R. Trim and fill: A simple funnel-plot-based method of testing and adjusting for publication bias in meta-analysis. Biometrics. 2000 Jun;56(2):455-63.

17. Yilmaz G, Akca A, Aydin N. Enhanced recovery after surgery (ERAS) versus conventional postoperative care in patients undergoing abdominal hysterectomies. Ginekol Pol. 2018;89(7):351-6.

18. Akca A, yilmAz G. Enhanced recovery after surgery (ERAS) improves SF-12 scores in patients undergoing gynecologic surgery: "positive butterfly effect". Acta Medica Mediterranea. 2019;35(4):2277-82.

19. Yilmaz G, Akca A, Kiyak H, Salihoglu Z. Comparison of enhanced recovery protocol with conventional care in patients undergoing minor gynecologic surgery. Wideochir Inne Tech Maloinwazyjne. 2020 Mar;15(1):220-6.

20. Marx C, Rasmussen T, Jakobsen DH, Ottosen C, Lundvall L, Ottesen B, et al. The effect of accelerated rehabilitation on recovery after surgery for ovarian malignancy. Acta Obstet Gynecol Scand. 2006;85(4):48892.

21. Sjetne IS, Krogstad U, Odegard S, Engh ME. Improving quality by introducing enhanced recovery after surgery in a gynaecological department: consequences for ward nursing practice. Qual Saf Health Care. 2009 Jun;18(3):236-40. 
22. Dickson E, Argenta PA, Reichert JA. Results of introducing a rapid recovery program for total abdominal hysterectomy. Gynecol Obstet Invest. 2012;73(1):21-5.

23. Kalogera E, Bakkum-Gamez JN, Jankowski CJ, Trabuco E, Lovely JK, Dhanorker S, et al. Enhanced recovery in gynecologic surgery. Obstet Gynecol. 2013 Aug;122(2 Pt 1):319-28.

24. de Groot JJ, van Es LE, Maessen JM, Dejong CH, Kruitwagen RF, Slangen BF. Diffusion of Enhanced Recovery principles in gynecologic oncology surgery: is active implementation still necessary? Gynecologic oncology. 2014 Sep;134(3):570-5.

25. Reif P, Drobnitsch T, Aigmuller T, Laky R, Ulrich D, Haas J, et al. The Decreasing Length of Hospital Stay following Vaginal Hysterectomy: 2011-2012 vs. 1996-1997 vs. 1995-1996. Geburtshilfe Frauenheilkd. 2014 May;74(5):449-53.

26. Wijk L, Franzen K, Ljungqvist O, Nilsson K. Implementing a structured Enhanced Recovery After Surgery (ERAS) protocol reduces length of stay after abdominal hysterectomy. Acta Obstet Gynecol Scand. 2014 Aug;93(8):749-56.

27. Yoong W, Sivashanmugarajan V, Relph S, Bell A, Fajemirokun E, Davies T, et al. Can enhanced recovery pathways improve outcomes of vaginal hysterectomy? Cohort control study. J Minim Invasive Gynecol. 2014 Jan-Feb;21(1):83-9.

28. Miller EC, McIsaac DI, Chaput A, Antrobus J, Shenassa H, Lui A. Increased postoperative day one discharges after implementation of a hysterectomy enhanced recovery pathway: a retrospective cohort study. Can J Anaesth. 2015 May;62(5):451-60.

29. Chapman JS, Roddy E, Ueda S, Brooks R, Chen LL, Chen LM. Enhanced Recovery Pathways for Improving Outcomes After Minimally Invasive Gynecologic Oncology Surgery. Obstet Gynecol. 2016 Jul;128(1):138-44.

30. Modesitt SC, Sarosiek BM, Trowbridge ER, Redick DL, Shah PM, Thiele RH, et al. Enhanced Recovery Implementation in Major Gynecologic Surgeries: Effect of Care Standardization. Obstet Gynecol. 2016 Sep;128(3):457-66.

31. Myriokefalitaki E, Smith M, Ahmed AS. Implementation of enhanced recovery after surgery (ERAS) in gynaecological oncology. Arch Gynecol Obstet. 2016 Jul;294(1):137-43.

32. Lambaudie E, de Nonneville A, Brun C, Laplane C, N'Guyen Duong L, Boher JM, et al. Enhanced recovery after surgery program in Gynaecologic Oncological surgery in a minimally invasive techniques expert center. BMC Surg. 2017 Dec 28;17(1):136.

33. Bergstrom JE, Scott ME, Alimi Y, Yen TT, Hobson D, Machado KK, et al. Narcotics reduction, quality and safety in gynecologic oncology surgery in the first year of enhanced recovery after surgery protocol implementation. Gynecologic oncology. 2018 Jun;149(3):554-9.

34. Bisch SP, Wells T, Gramlich L, Faris P, Wang X, Tran DT, et al. Enhanced Recovery After Surgery (ERAS) in gynecologic oncology: System-wide implementation and audit leads to improved value and patient outcomes. Gynecologic oncology. 2018 Oct;151(1):117-23.

35. Boitano TKL, Smith HJ, Rushton T, Johnston MC, Lawson P, Leath CA, 3rd, et al. Impact of enhanced recovery after surgery (ERAS) protocol on gastrointestinal function in gynecologic oncology patients undergoing laparotomy. Gynecologic oncology. 2018 Nov;151(2):282-6.

36. Carter-Brooks CM, Du AL, Ruppert KM, Romanova AL, Zyczynski HM. Implementation of a urogynecology-specific enhanced recovery after surgery (ERAS) pathway. Am J Obstet Gynecol. 2018 Nov;219(5):495 e1- e10.

37. de Groot JJA, Maessen JMC, Dejong CHC, Winkens B, Kruitwagen R, Slangen BFM, et al. Interdepartmental Spread of Innovations: A Multicentre Study of the Enhanced Recovery After Surgery Programme. 
World J Surg. 2018 Aug;42(8):2348-55.

38. Mendivil AA, Busch JR, Richards DC, Vittori H, Goldstein BH. The Impact of an Enhanced Recovery After Surgery Program on Patients Treated for Gynecologic Cancer in the Community Hospital Setting. International journal of gynecological cancer : official journal of the International Gynecological Cancer Society. 2018 Mar;28(3):581-5.

39. Meyer LA, Lasala J, Iniesta MD, Nick AM, Munsell MF, Shi Q, et al. Effect of an Enhanced Recovery After Surgery Program on Opioid Use and Patient-Reported Outcomes. Obstet Gynecol. 2018 Aug;132(2):28190 .

40. Trowbridge ER, Evans SL, Sarosiek BM, Modesitt SC, Redick DL, Tiouririne M, et al. Enhanced recovery program for minimally invasive and vaginal urogynecologic surgery. Int Urogynecol J. 2018 Feb;30(2):313-21.

41. Agarwal R, Rajanbabu A, th PVN, Goel G, Madhusudanan L, U GU. A prospective study evaluating the impact of implementing the ERAS protocol on patients undergoing surgery for advanced ovarian cancer. International journal of gynecological cancer : official journal of the International Gynecological Cancer Society. 2019 Mar;29(3):605-12.

42. Ferrari F, Forte S, Sbalzer N, Zizioli V, Mauri M, Maggi C, et al. Validation of an enhanced recovery after surgery protocol in gynecological surgery: an Italian randomized study. American journal of obstetrics and gynecology. $2020 \mathrm{Jul} 8$.

43. Peters A, Siripong N, Wang L, Donnellan NM. Enhanced recovery after surgery outcomes in minimally invasive nonhysterectomy gynecologic procedures. Am J Obstet Gynecol. 2020 Feb 20.

44. Kalogera E, Glaser GE, Kumar A, Dowdy SC, Langstraat CL. Enhanced Recovery after Minimally Invasive Gynecologic Procedures with Bowel Surgery: A Systematic Review. J Minim Invasive Gynecol. 2019 Feb;26(2):288-98.

45. Ljungqvist O, Young-Fadok T, Demartines N. The History of Enhanced Recovery After Surgery and the ERAS Society. J Laparoendosc Adv Surg Tech A. 2017 Sep;27(9):860-2.

46. Scheib SA, Thomassee M, Kenner JL. Enhanced Recovery after Surgery in Gynecology: A Review of the Literature. J Minim Invasive Gynecol. 2019 Feb;26(2):327-43.

47. Group EC. The Impact of Enhanced Recovery Protocol Compliance on Elective Colorectal Cancer Resection: Results From an International Registry. Ann Surg. 2015 Jun;261(6):1153-9.

48. Nelson G, Ramirez PT, Ljungqvist O, Dowdy SC. Enhanced Recovery Program and Length of Stay After Laparotomy on a Gynecologic Oncology Service: A Randomized Controlled Trial. Obstet Gynecol. 2017 Jun;129(6):1139.

49. Iniesta MD, Lasala J, Mena G, Rodriguez-Restrepo A, Salvo G, Pitcher B, et al. Impact of compliance with an enhanced recovery after surgery pathway on patient outcomes in open gynecologic surgery. International journal of gynecological cancer : official journal of the International Gynecological Cancer Society. 2019 Nov;29(9):1417-24.

50. Wijk L, Udumyan R, Pache B, Altman AD, Williams LL, Elias KM, et al. International validation of Enhanced Recovery After Surgery Society guidelines on enhanced recovery for gynecologic surgery. Am J Obstet Gynecol. 2019 Sep;221(3):237.e1-.e11.

51. Altman AD, Nelson GS. The Canadian Gynaecologic Oncology Perioperative Management Survey: Baseline Practice Prior to Implementation of Enhanced Recovery After Surgery (ERAS) Society Guidelines. Journal of obstetrics and gynaecology Canada : JOGC = Journal d'obstetrique et gynecologie du Canada : JOGC. 2016 Dec;38(12):1105-9.e2.

52. Piovano E, Ferrero A, Zola P, Marth C, Mirza MR, Lindemann K. Clinical pathways of recovery after surgery for advanced ovarian/tubal/peritoneal cancer: an NSGO-MaNGO international survey in collabora- 
tion with AGO-a focus on surgical aspects. International journal of gynecological cancer : official journal of the International Gynecological Cancer Society. 2019 Jan;29(1):181-7.

53. Lu D, Wang X, Shi G. Perioperative enhanced recovery programmes for gynaecological cancer patients. Cochrane Database Syst Rev. 2015 Mar 19(3):CD008239.

54. Cui L, Shi Y, Zhang GN. Fast-track surgery after gynaecological oncological surgery: study protocol for a prospective randomised controlled trial. Trials. 2016 Dec 15;17(1):597.

55. Senturk JC, Kristo G, Gold J, Bleday R, Whang E. The Development of Enhanced Recovery After Surgery Across Surgical Specialties. J Laparoendosc Adv Surg Tech A. 2017 Sep;27(9):863-70.

56. de Groot JJA, van Es LE, Maessen JM, Dejong CH, Kruitwagen RF, Slangen BF. Diffusion of Enhanced Recovery principles in gynecologic oncology surgery: is active implementation still necessary? Gynecologic oncology. 2014 Sep;134(3):570-5.

57. Acter T, Uddin N, Das J, Akhter A, Choudhury TR, Kim S. Evolution of severe acute respiratory syndrome coronavirus 2 (SARS-CoV-2) as coronavirus disease 2019 (COVID-19) pandemic: A global health emergency. Sci Total Environ. 2020 Aug 15;730:138996.

58. Lai CC, Shih TP, Ko WC, Tang HJ, Hsueh PR. Severe acute respiratory syndrome coronavirus 2 (SARS-CoV-2) and coronavirus disease-2019 (COVID-19): The epidemic and the challenges. Int J Antimicrob Agents. 2020 Mar;55(3):105924.

59. Liang W, Guan W, Chen R, Wang W, Li J, Xu K, et al. Cancer patients in SARS-CoV-2 infection: a nationwide analysis in China. Lancet Oncol. 2020 Mar;21(3):335-7.

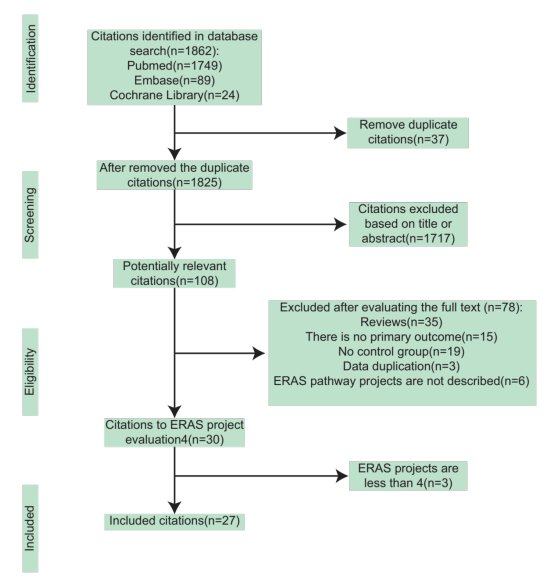




\begin{tabular}{|c|c|c|}
\hline Study ID & $\operatorname{SMD}(95 \% \mathrm{Cl})$ & Weight \% \\
\hline Marx C 2006 & $-0.23(-0.56,0.10)$ & 4.11 \\
\hline Sjetne IS 2009 & $-2.04(-2.51,-1.58)$ & 3.53 \\
\hline Dickson E 2012 & $-0.88(-1.17,-0.58)$ & 4.25 \\
\hline Kalogera E 2013 & $-0.33(-0.51,-0.15)$ & 4.66 \\
\hline Reif P 2014 & $-1.25(-1.58,-0.92)$ & 4.12 \\
\hline Wijk L 2014 & $-0.22(-0.50,0.06)$ & 4.32 \\
\hline Yoong W 2014 & $-1.82(-2.29,-1.35)$ & 3.52 \\
\hline Miller EC 2015 & $-0.89(-1.16,-0.61)$ & 4.33 \\
\hline Chapman JS 2016 & $-1.20(-1.55,-0.85)$ & 4.04 \\
\hline Modesitt SC 2016 & $-0.43(-0.57,-0.30)$ & 4.79 \\
\hline Myriokefalitaki E 2016 & $-0.66(-0.94,-0.37)$ & 4.29 \\
\hline Lambaudie E 2017 & $-0.35(-0.62,-0.07)$ & 4.32 \\
\hline Bergstrom JE 2018 & $0.00(-0.24,0.24)$ & 4.45 \\
\hline Bisch SP 2018 & $-0.40(-0.59,-0.21)$ & 4.63 \\
\hline Boitano TKL 2018 & $-0.51(-0.71,-0.30)$ & 4.59 \\
\hline Carter-Brooks CM 2018 & $-1.11(-1.37,-0.84)$ & 4.38 \\
\hline Mendivil A A 2018 & $-0.53(-0.83,-0.23)$ & 4.24 \\
\hline Meyer LA 2018 & $-0.11(-0.36,0.13)$ & 4.46 \\
\hline Trowbridge ER 2018 & $-0.26(-0.55,0.03)$ & 4.28 \\
\hline Yilmaz G 2018 & $-0.70(-1.21,-0.18)$ & 3.33 \\
\hline Agarwal R 2019 & $-0.74(-1.17,-0.32)$ & 3.70 \\
\hline Akca A 2019 & $-0.47(-0.89,-0.05)$ & 3.72 \\
\hline Ferrari F 2020 & $-0.75(-1.06,-0.43)$ & 4.19 \\
\hline Yilmaz G 2020 & $-1.12(-1.54,-0.71)$ & 3.75 \\
\hline Overall $(1-$ squared $=88.2 \%, p=0.000)$ & $-0.68(-0.84,-0.52)$ & 100.00 \\
\hline
\end{tabular}

B

\begin{tabular}{|c|c|c|}
\hline Study ID & SMD $(95 \% \mathrm{Cl})$ & Weight \% \\
\hline Marx C 2006 & $-0.33(-0.66,0.00)$ & 17.21 \\
\hline Kalogera E 2013 & $-0.25(-0.42,-0.07)$ & 22.52 \\
\hline de Groot JJA 2014 & $-0.95(-1.35,-0.54)$ & 14.74 \\
\hline Bisch SP 2018 & $-0.30(-0.49,-0.11)$ & 22.17 \\
\hline de Groot JJA 2018 & $0.02(-0.13,0.17)$ & 23.37 \\
\hline Overall $(1-$ squared $=82.6 \%, p=0.000)$ & $-0.31(-0.55,-0.07)$ & 100.00 \\
\hline NOTE: Weights are from random effects analysis & & \\
\hline
\end{tabular}

C

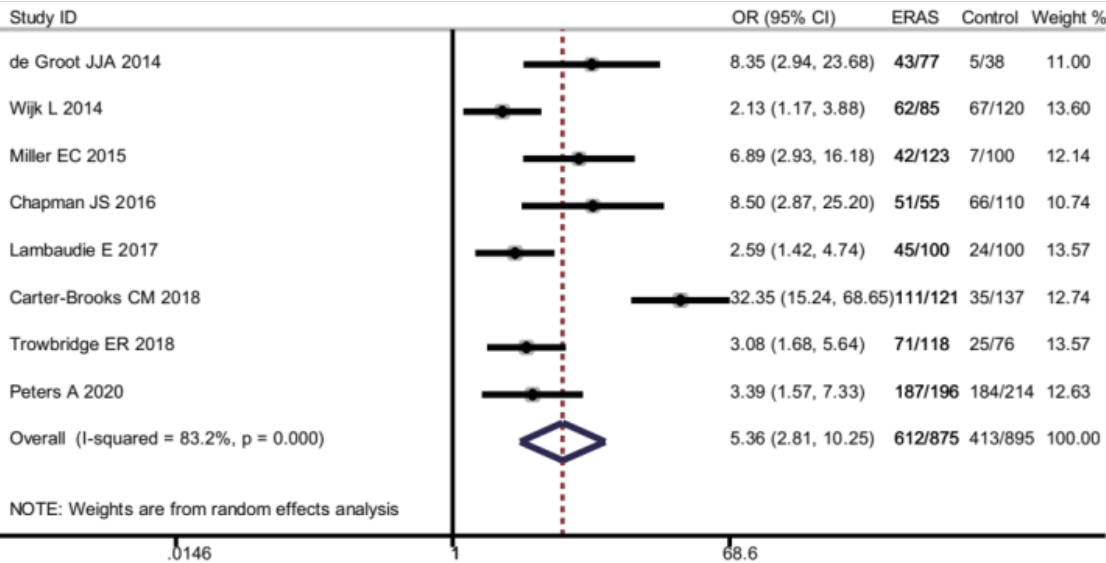



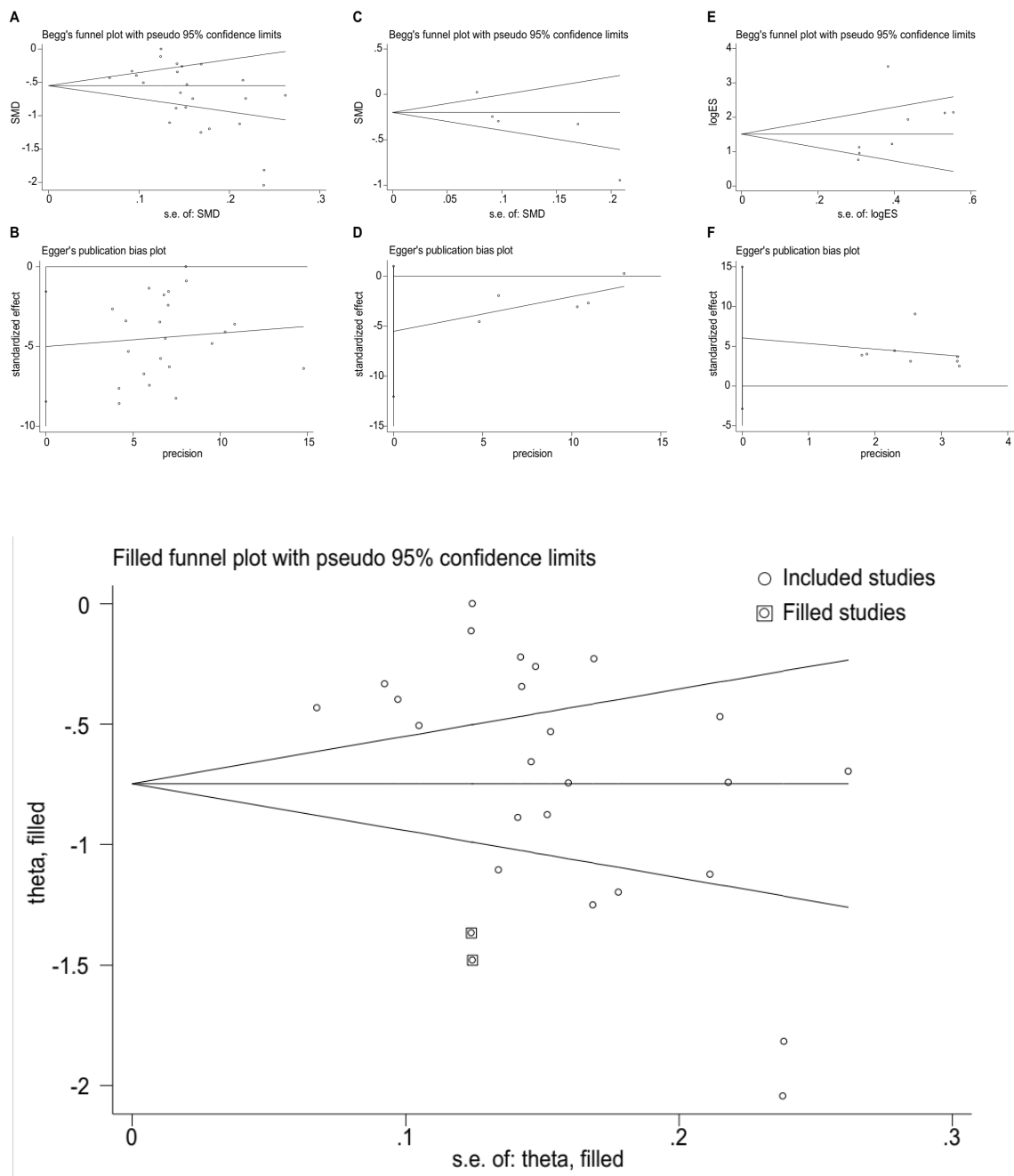

\section{Hosted file}

Table.1.docx available at https://authorea.com/users/352432/articles/476697-enhancedrecovery-after-surgery-eras-in-gynaecology-a-meta-analysis

\section{Hosted file}

Table.2.docx available at https://authorea.com/users/352432/articles/476697-enhancedrecovery-after-surgery-eras-in-gynaecology-a-meta-analysis

\section{Hosted file}

Table.3.docx available at https://authorea.com/users/352432/articles/476697-enhancedrecovery-after-surgery-eras-in-gynaecology-a-meta-analysis

\section{Hosted file}

Table.4.docx available at https://authorea.com/users/352432/articles/476697-enhancedrecovery-after-surgery-eras-in-gynaecology-a-meta-analysis 